Abstract 419 Table 1 Patient Characteristics (at incident SLE hospitalisation) and study outcomes.

\begin{tabular}{|c|c|c|c|}
\hline & $\begin{array}{l}\text { SLE as reason for } \\
\text { admission } \\
(n=1510)\end{array}$ & $\begin{array}{l}\text { Matched } \\
\text { Controls } \\
(n=1320)\end{array}$ & Rort-test \\
\hline & IoCTorn(\%) & MoCTorn $(\%)$ & (p-ralue) \\
\hline Age & $45.15 \pm 19.44$ & $50.07 \pm 21.66$ & $<0.001$ \\
\hline Female & $1172(77.3 \%)$ & $1031(78.1 \%)$ & 0.611 \\
\hline Indigenous & $107(7.1 \%)$ & $21(1.60 \%)$ & 40.001 \\
\hline Length of Stay (darys) & $5([Q R 1,11)$ & $1([Q R 1.5)$ & 40.001 \\
\hline Privately Insured & $176(31.5 \%)$ & $325(52.3 \%)$ & $\varangle 0.001$ \\
\hline Diagnosed nith Ischaemic Heart Disease & $46(3.036)$ & $28(2.1 \%)$ & 0.128 \\
\hline Diagnosed with a Cerebral Ischaemic Disease & $16(1.1 \%)$ & $8(0.6 \%)$ & 0.193 \\
\hline Disgnosed rith Hypertension & $165(10.940)$ & $71(5.4 \%)$ & $\diamond 0.001$ \\
\hline Diagnosed with an Atherosclerotic Condition & $54(3.690)$ & $11(0.8 \%)$ & $\varangle 0.001$ \\
\hline Diagnosed nith a Kidner Disorder & $308(20.3 \%)$ & $13(1.096)$ & $<0.001$ \\
\hline Diagnosed with a Thrombotic Disorder & $10(0.7 \%)$ & $5(0.49)$ & 0304 \\
\hline Patientdied during the hospital admission & $37(2.496)$ & $17(1.39 \%)$ & 40.001 \\
\hline \multicolumn{4}{|c|}{ MoCT:Measure of Central Tendency, Le mean \pm standard deviabon or median (nterguartle range) } \\
\hline
\end{tabular}

421

ASSESSING THE QUALITY, RELIABILITY AND READABILITY OF ONLINE HEALTH INFORMATION REGARDING SYSTEMIC LUPUS ERYTHEMATOSUS (SLE)

${ }^{1} \mathrm{M}$ Reynolds*, ${ }^{1} \mathrm{~A}$ Hoi, ${ }^{1,2}$ R Buchanan. 'Austin Health, Rheumatology, Heidelberg, Australia; ${ }^{2}$ University of Melbourne, Department of Medicine, Melbourne, Australia

\subsection{6/lupus-2017-000215.421}

Background and aims Systemic lupus erythematosus (SLE) is a complex multi-system autoimmune disorder. Patients frequently access the internet to increase their knowledge about the condition.

We assessed the quality, reliability and readability of online sites relating to SLE.

Methods The search-phrase 'systemic lupus erythematosus' was used with the three most commonly accessed internet search engines (Google, Bing and Yahoo) to identify websites. The first 25 hits (excluding duplicates and excluded websites) for each search were assessed for quality using the DISCERN instrument (scores 15-80 points), reliability using the JAMA benchmarks (assessing authorship, attribution of references, currency (date of posted content) and disclosure of conflict of interest) and readability using the Gunning-FOG index (ideal score 7-8).

Results There was significant concordance between the hits returned from each search engine with 26 unique websites identified. The average DISCERN score was 47.7 (SD 13.2), and ranged from 19-75. Websites that appeared earlier in searches did not have higher DISCERN scores (Pearson correlation -0.16).

Currency was present in 50.0\%, appropriate authorship in $46.2 \%$ and attribution of references and disclosure of interest in only $15.4 \%$ of websites.

The average readability of the websites was 9.3 (SD 3.4) using the FOG index.

Conclusions The overall quality of online health information relating to SLE is only fair. 


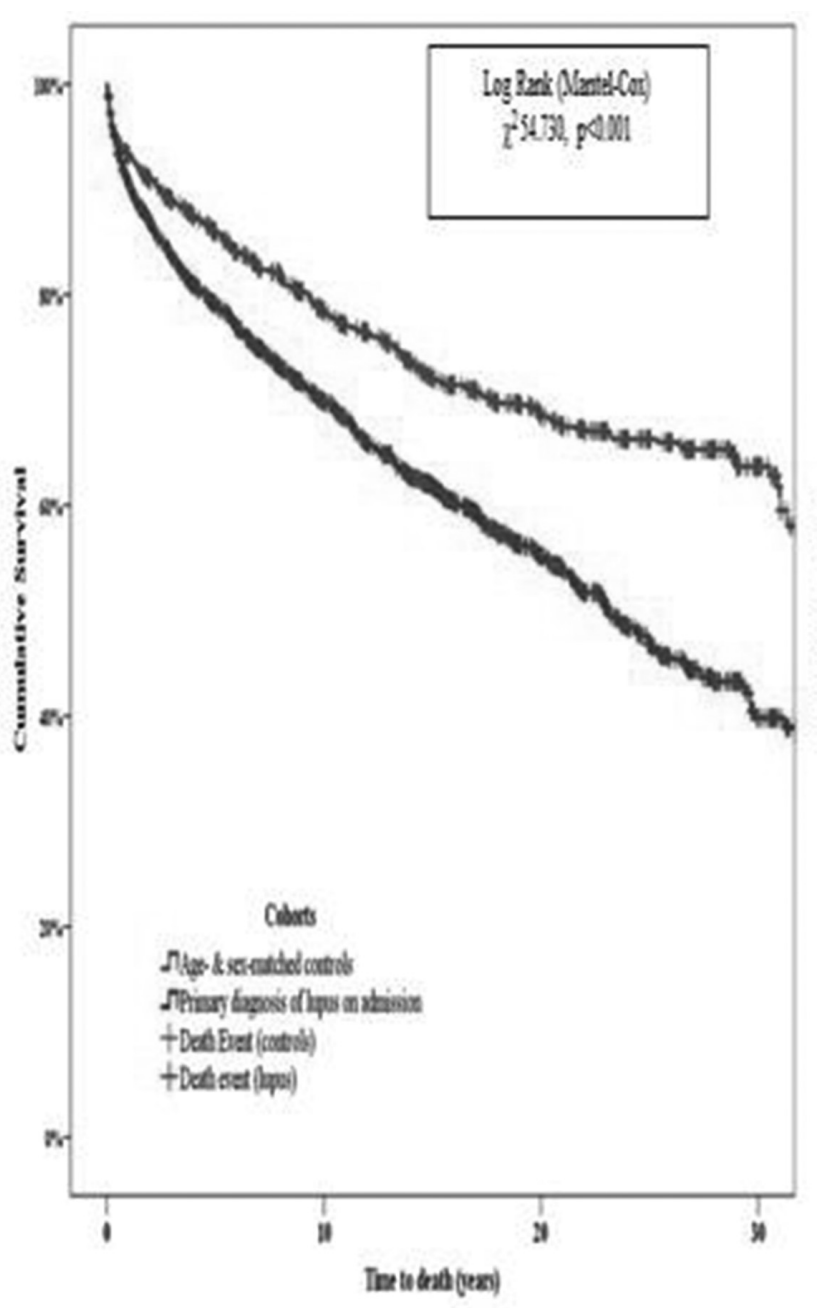

Abstract 419 Figure 1 Kalpan-Meier survival analysis of all-cause motality for SLE patients and age- and sex-matched controls (free of rheumatic disease) from index hospitalisation.

Reliability as measured by the JAMA benchmarks was average to poor.

The readability is higher than recommended for the general public, which may limit understanding.

This assessment highlights the need for clinicians to provide patients with alternative sources of high quality information regarding SLE.

\section{ANTI-RNP/SM ANTIBODIES PLUS LUPUS ANTICOAGULANT AS RISK FACTOR FOR THROMBOSIS IN PATIENTS WITH SYSTEMIC LUPUS ERYTHEMATOSUS}

${ }^{1}$ MC Zamora-Medina*, ${ }^{1}$ A Hinojosa-Azaola, ${ }^{2}$ AG Vargas-Ruiz, 'J Sanchez-Guerrero, ${ }^{1} \mathrm{C}$ Nunez-Alvarez, ${ }^{1} \mathrm{~J}$ Romero-Diaz. ${ }^{1}$ Instituto Nacional de Ciencias Medicas y Nutricion S.Z., Immunology and Rheumatology, Mexico city, Mexico; ${ }^{2}$ Instituto Nacional de Ciencias Medicas y Nutricion S.Z., Haematology, Mexico city, Mexico

\subsection{6/lupus-2017-000215.422}

Background and aims Thrombosis still remains as main cause of morbidity and mortality in SLE patients. Results from a recent study identified a strong association of anti-RNP/Sm abs+LA with thrombosis. We aimed to validate this association

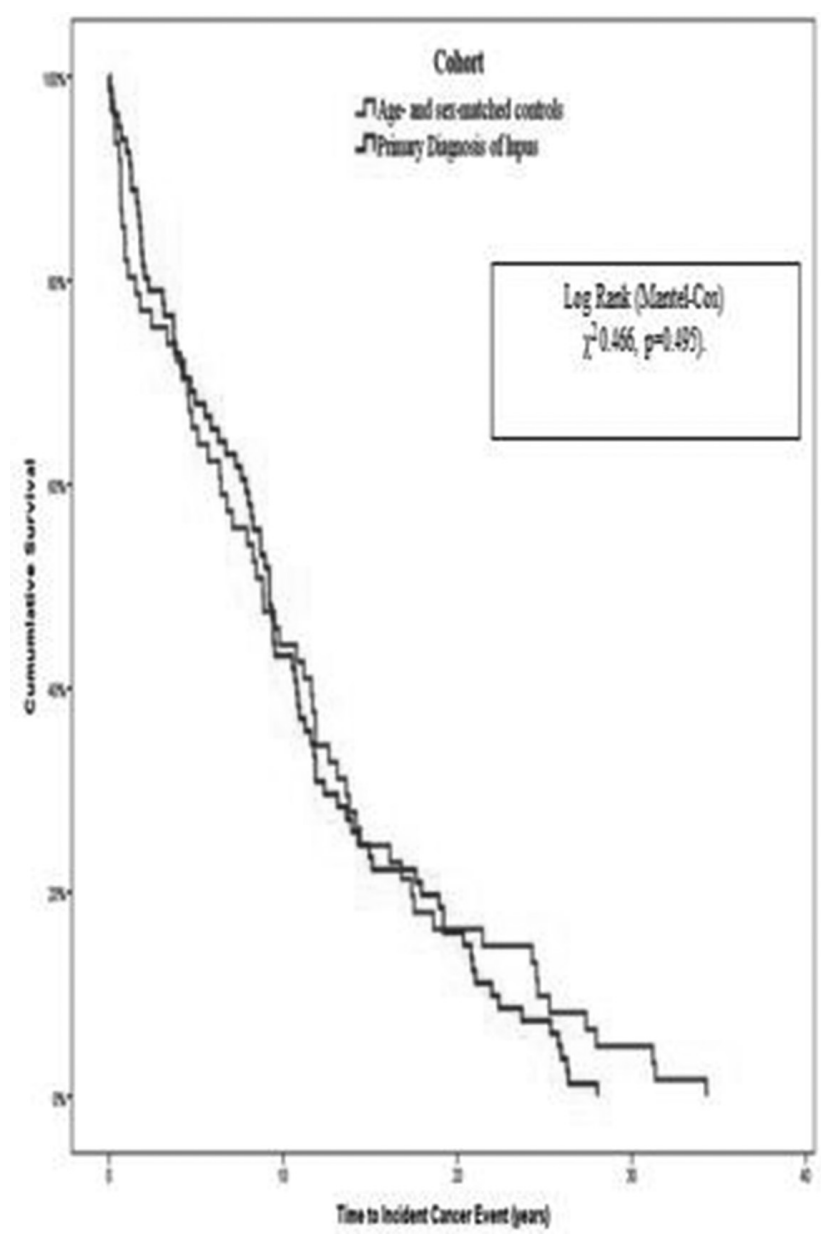

Abstract 419 Figure 2 Kalpan-Meier survival analysis of cancer outcomes for SLE patients and age- \& sex-matched controls (free of rheumatic disease conditions) from incident hospitalisation.

Methods We studied 63 SLE patients (>4 criteria/ACR) with confirmed history of thrombosis after SLE diagnosis. As controls, 63 SLE patients without thrombosis, matched by age, gender and lupus duration were included. Disease characteristics, medication, traditional risk factors for thrombosis and thrombotic event information were retrieved from clinical files. A blood sample was drawn to determine anti-cardiolipin (IgG/IgM), antiß2-GP1 (IgG/IgM), LA, anti-RNP/Sm and anti$\mathrm{Sm}$ antibodies. Sensitivity, specificity, positive and negative predictive values (PPV, NPV) and Likelihood Ratios (LR) were calculated.

Results One hundred and twenty six SLE patients were studied. Cases and controls were similar in age, gender and disease duration $(p=N S)$. There were no differences in the prevalence of traditional risk factor for thrombosis between cases and controls $(p=N S)$. Among patients with thrombosis a higher frequency of anti-RNP/Sm ( $83 \%$ vs $62 \%, \mathrm{p}<0.001)$, LA (62\% vs $19 \%, \mathrm{p}<0.001)$, aPL triple marker $(17 \%$ vs $2 \%$, $\mathrm{p}=0.04)$ and anti-RNP/Sm+AL combination (52\% vs $14 \%$, $\mathrm{p}<0.001$ ) was observed. The combination of anti-RNP/Sm + AL showed a sensitivity 52\%, specificity $86 \%$, PPV 78\% and NPV 65\%, positive LR 3.67 (IC 95\% 1.92-7.04) and negative LR 0.55 (IC 95\% 0.42-0.74).

Conclusions This study confirmed that anti-RNP/Sm+LA association represents a risk factor for thrombosis in SLE patients. 